\title{
Two types of fog shapes above a cold evaporating liquid
}

\author{
A. Feasson*, A. Taihi, and B. Correge \\ Univ. Paris-Diderot, Université de Paris, Bâtiment Condorcet, 4 rue Elsa Morante, 75013 Paris, France
}

Received: 26 August 2020 / Accepted: 12 January 2021

\begin{abstract}
When liquid nitrogen is poured into a mug, a mist forms above. This article explores the influence of air humidity on the cloud and the formation of these boundaries in a controlled environment. We have identified that both homogeneous and heterogeneous nucleations occur during their formation. We highlight two types of ice clouds that differ only in the level of air humidity. Indeed, there is a critical humidity level at which one goes from a banded cloud to another without lower limit, extending to the cold liquid. We argue that this critical humidity level is related to the nitrogen flux.
\end{abstract}

Keywords: Cold cloud shapes / evaporating liquid / humidity / nucleation / inverted temperature gradient.

\section{Introduction}

This study is about the formation of a fog above liquid nitrogen. A fog or a cloud is composed of hydrometeors [1] that can grow in a supersaturated environment. Hydrometeors in our case are water or ice particles that form in the atmosphere or near the ground by condensation. They become visible as a cloud when they become big enough to scatter light [1]. Some previous studies have examined the conditions for fog formation near cool surfaces [2] but virtually none of them involve the evaporation of an extremely cold liquid, here nitrogen. This boundary condition is different from what we usually have in clouds and could involve other mechanisms for its shape. This is one of the reasons why we became interested in studying it. In atmospheric physics there is a type of cloud, the cold cloud that can be composed of either just ice or ice and supercooled droplets. To form a cold cloud, the temperature must be below $0{ }^{\circ} \mathrm{C}[3]$. The clouds produced would be like cold clouds. However, in this case, there is a huge difference with the atmospheric cloud: the temperature gradient is reversed, i.e. the temperature increases with height. So there is a stable stratification in the gaseous phase.

After liquid nitrogen is poured into a container, it starts to evaporate at a temperature around $77 \mathrm{~K}$ and a flat fog forms over it. But we quickly notice what appears to be, in our controlled environment, two types of clouds depending of the humidity. The first is a banded cloud with an upper and lower boundary, and the second is a cloud without a lower limit that will be called an extended cloud (see Fig. 1).

\footnotetext{
*e-mail: a.feasson1@gmail.com
}

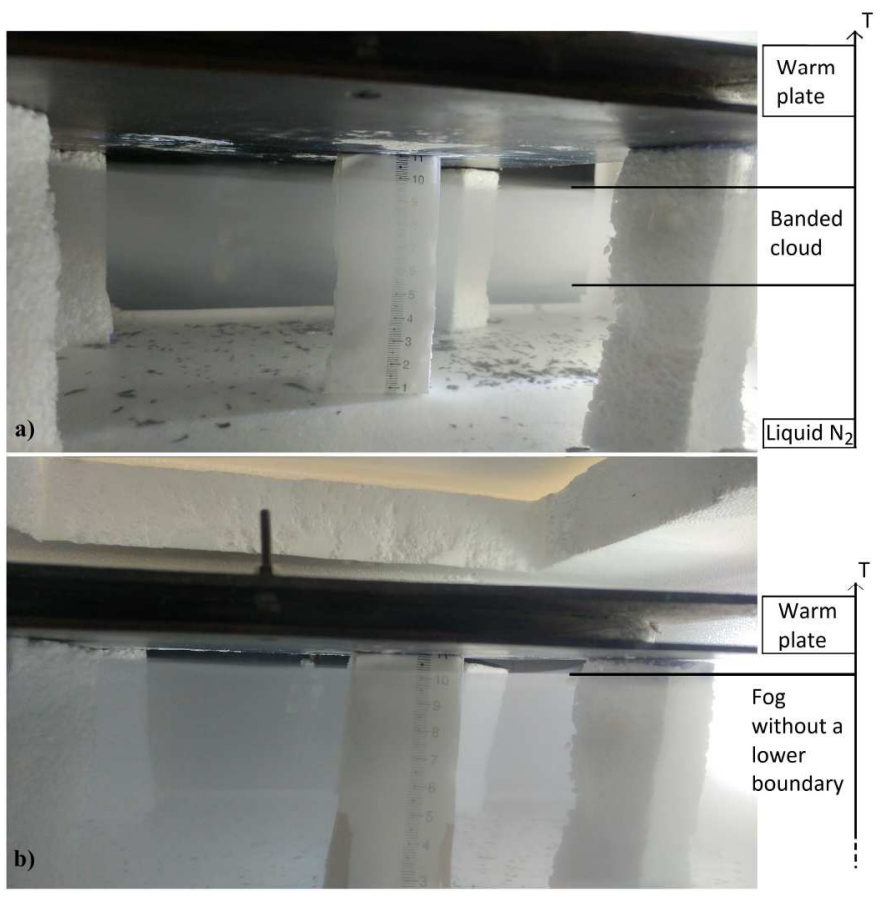

Fig. 1. Pictures of both type of clouds: (a) the banded cloud, (b) the extended cloud. A central pillar scales, in centimetres, the space between the liquid nitrogen bath and the plate. Schematic diagrams with the direction of the temperature gradient are next to the images.

Our objective is therefore to determine the mechanisms that influence the height, thickness of the cloud and the appearance of its boundaries. We will also look at its constituents and the process by which the drops are formed. 


\section{Method}

\subsection{The experimental setup}

The experimental setup consists of a rectangular box made of expanded polystyrene foam used for its resistance to cold and its low thermal conductivity (see Fig. 2). For observation, this enclosure has a double plexiglass glazing on one side to prevent forming mist and frost on the glass (see dead end section). An opening in the box's upper side allows us to pour liquid nitrogen to cover the entire bottom of the box. Three small holes are made to supply humid air and for the water circuit that thermalizes the hot plate. A vertical slit has also been made to illuminate the inside of the box with a LED light source in order to observe the experiments and read the measurements. The observation chamber rests on a balance so as to put the same quantity of liquid nitrogen for each measurement run $(500 \mathrm{~g})$, and to monitor the quantity of remaining liquid nitrogen.

Two heat sources separated by $11 \mathrm{~cm}$ were used to create a constant temperature gradient inside the box. The first source is a cold source at the bottom of the box which consists of liquid nitrogen at temperature $T_{c}$ around $77 \mathrm{~K}$. The second is a hot plate made of copper near the top of the box installed over pillars. One of these pillars is graduated so as to measure the height of the fog. The plate is internally supplied with hot water at a controlled temperature of $T_{h}=30^{\circ} \mathrm{C}$.

A bubbler system (see Fig. 2) was used in order to inject humid air inside the box: pressurized air bubbles through hot water placed in a jar, then the humid air formed is recovered and transferred into the box using a pipe. The rate of humid air flow was controlled by a tap, and was kept low so as not to perturb the fog and the temperature gradient. Each time liquid nitrogen was poured, we did not started the measures until the fog was definitely homogeneous inside the box.

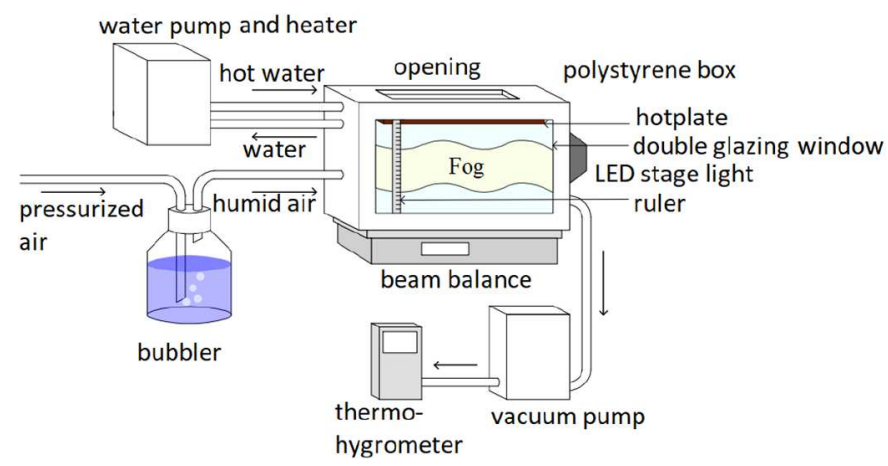

Fig. 2. Scheme of the experimental setup (see text). The bottom of the polystyrene box is covered by liquid nitrogen.

To verify the constancy of the temperature gradient, a combined thermo-hygrometer was inserted inside the box at different heights (see Fig. 3). With this latter, we could also evaluate relative humidity. The lower limit temperature of the thermometer is $-200^{\circ} \mathrm{C}$ and that of the hygrometer is $-20^{\circ} \mathrm{C}$. However, the temperatures inside the box can reach nearly $-200^{\circ} \mathrm{C}$. That's why it was possible to measure directly the temperature but not the humidity. We created a system using a vacuum pump to sample the air of our box in order to measure its hygrometry outside the box. The vacuum pump consists mainly of a vacuum bell jar, a pump and a pipe that ends with a needle. The hygrometer-probe was put inside the vacuum bell and its digital display was put outside the bell jar. All the fittings were sealed tight to avoid leaks. For each measurement, the needle was entered at several heights inside the box, the pump was turned on and the air was therefore collected and transferred through the hose into the vacuum chamber. Thereafter, the air was warmed to ambient temperatures and we could determine its moisture content. The sampling did not, a priori, have any influence on the cloud.

To determine the upper and the lower bounds of the fog, we used the graduated pillar and a camera placed in front of the glass. A picture was taken each time we collected the humid air in order to know the cloud's height and thus its temperature.

\subsection{The theoretical framework}

The air we breathe contains typically about $0.5 \%$ of water vapour. In contact with liquid nitrogen, which has a very low temperature (around $77 \mathrm{~K}$ ), this water vapour condenses and turns into droplets or ice crystals suspended in the air. This condensation is observed in the form of a fog.

Condensation of water vapour relies on the saturation of the air in water vapour, which strongly depends on temperature. The quantity of water vapour contained in the air is known as partial pressure $P_{i}$. At a temperature below the boiling point, air can carry a certain definite amount of water vapour. This partial pressure limit, known as equilibrium vapour pressure $P_{\text {sat }}$, varies as a function of temperature according to the Clausius-Clapeyron relation:

$$
\frac{d P_{\mathrm{sat}}(T)}{d T}=\frac{P_{\mathrm{sat}} \triangle H}{R T^{2}}
$$

Assuming that the enthalpy of vaporization $\triangle H$ does not vary with temperature, the integrated form becomes:

$$
P_{\text {sat }}(T)=A \exp \left(\frac{-\triangle H}{R T}\right)
$$

with $A$ - constant, $P_{\text {sat }}(\mathrm{T})$ - equilibrium vapour pressure, $\triangle H$ - enthalpy of vaporization of water and $R$ - ideal gas constant.

The higher the temperature, the higher the equilibrium vapour pressure and vice versa.

The ratio between the partial pressure of water vapour $P_{i}$ and its equilibrium pressure $P_{\text {sat }}$ defines the relative humidity:

$$
R H=\frac{P_{i}}{P_{\text {sat }}} \times 100 \% \text {. }
$$


Condensation happens when this ratio is equal to or greater than $100 \%$ (dew point): the surplus of water vapour condenses into droplets. The dew point is exceeded in two ways:

The first case happens at a fixed temperature, when the partial pressure of water vapour $P_{i}$ increases to be equal to or greater than its equilibrium pressure $P_{\text {sat }}$.

The second one occurs when the partial pressure of water vapour $P_{i}$ is sustained constant but the temperature decreases inducing a decrease of the equilibrium pressure of water vapour $P_{\text {sat }}$.

In some conditions, $\mathrm{RH}$ can achieve greater values than $100 \%$ without condensation. This phenomenon is called supersaturation [4].

\subsection{Nucleation processes}

The initialization of condensation is a kinetic phenomenon which needs to overcome a barrier of energy through a process which is called nucleation.

Nucleation is the aggregation of water molecules around condensation nuclei forming the droplets. There are two types of nucleation depending on the sort of this nuclei: homogeneous and heterogeneous nucleations [4].

On the one hand, when the drop forms around a single initial water molecule, the nucleation is homogeneous. This process occurs only if the cohesion forces' work inside the developing drop exceeds the energy barrier necessary for the formation of a liquid-vapour interface or an ice-vapour interface, and balances the external pressure work as well. This type of nucleation requires extreme conditions of supersaturation [3,4]. Indeed, when the supersaturation increases the energy barrier becomes weaker, making nucleation more feasible. The radius at which the crystal or drop can continue to grow without collapsing is called the critical radius $r_{c}$ and can be defined by the equation below [5]:

$$
P_{n}=P_{\text {sat }, \text { ice }} \times \exp \left(\frac{2 \sigma V_{g}}{k T r_{c}}\right)
$$

with $P_{n}$ the partial pressure of water, $P_{\text {sat,ice }}$ the saturating pressure, $\sigma$ the surface tension and $V_{g}$ the volume of a water molecule.This equation can be rewritten with the relative humidity defined in equation (3):

$$
R H=100 \times \exp \left(\frac{2 \sigma V_{g}}{k T r_{c}}\right)
$$

This shows that the critical radius $r_{c}$ is a decreasing function of the relative humidity $\mathrm{RH}$. Therefore, as the relative humidity increases, the critical radius $r_{c}$ decreases and nucleation becomes easier. However, when $r_{c}$ is of the same order of magnitude as the size of the molecules that make up the crystal, the energy barrier becomes almost non-existent. Crystals develop without constraint. In our case, this limitation is around one or two water molecule sizes, namely $3.2 \AA$ and $6.4 \AA$.

On the other hand, heterogeneous nucleation happens when the drops form around impurities and dust particles present in the air. These factors decrease considerably the energy barrier of drop formation making this process easier to take place. This phenomenon is generally involved in cloud formation. However, we cannot exclude the occurrence of homogeneous nucleation, as it was reported by Rosner and Epstein [2] studying the fog above a cold surface. Thus, these phenomena will be studied as carefully as possible.

\subsection{Link between the height and the temperature}

Experimentally, we have imposed a constant gradient temperature by fixing the temperature of a heat source $11 \mathrm{~cm}$ above the bath of liquid nitrogen. Figure 3 shows that temperature increases linearly with height. The gradient constancy is thus verified.

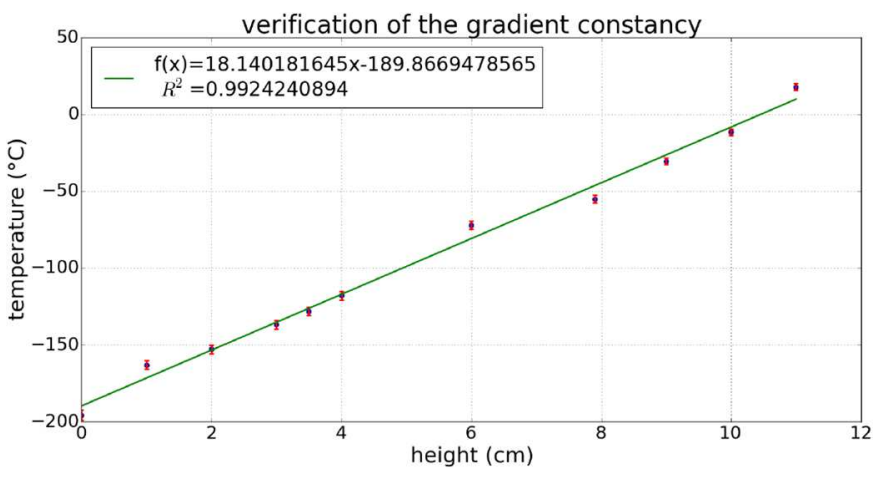

Fig. 3. Verification of temperature gradient: The temperature is proportional to the height above the liquid nitrogen bath. The temperature at $h=11 \mathrm{~cm}$ is imposed.

Assuming that the temperature gradient is constant and using boundary conditions:

$$
z_{u}=\frac{h \times\left(T_{u}-T_{c}\right)}{T_{h}-T_{c}}
$$

where $z_{u}$ : the upper height of the cloud. $T_{u}$ : the temperature at the upper limit of the cloud. $T_{h}$ : Hot boundary temperature. $T_{c}$ : cold boundary temperature (nearly $77 \mathrm{~K}$ ). $h$ : the height between the heat sources.

\subsection{Link between the height and the partial pressure of water vapour}

In a heterogeneous nucleation, we can consider that $P_{i} \simeq P_{\text {sat }}$ at the cloud top boundary and using ClausiusClapeyron relation (2), we find:

$$
T_{u}=\frac{\triangle H}{R \ln \left(\frac{A}{P_{i}}\right)}
$$

By combining equations (6) and (7), we obtain:

$$
z_{u}=\frac{\frac{\Delta H}{R \ln \left(\frac{A}{P_{i}}\right)}-T_{c}}{\frac{T_{h}-T_{c}}{h}}
$$

The upper boundary of the cloud should increase with the partial pressure of water vapour. 


\section{Results}

\subsection{Origin of the cloud}

The temperature $T_{i}$ of the cloud can be measured by taking a photo of it: the temperature gradient being linear, the height of a point gives its temperature. To measure the partial pressure $P_{i}$ of water vapour, a hygrometry measurement $\mathrm{RH}$ is made on the sample inside the vacuum chamber. However, this measurement is made at ambient temperature $T_{a}$, which is the temperature of the room. We denote $P_{\text {sat,a }}$ the saturated vapour pressure at temperature $T_{a}$. This pressure corresponds to the vapour/ice border of the state change diagram. It can be a partial pressure or, in the case of a pure body, the pressure of the gas. From equation (3), the perfect gas law for water vapour then gives the following relation:

$$
P_{i}=P_{\text {sat }, \mathrm{a}} \times \frac{R H}{100} \frac{T_{i}}{T_{a}} .
$$

By measuring the pressure and the temperature of the cloud, and by placing these points on the TemperaturePressure (TP) diagram of water (see Fig. 4), we realise that all the measurements are in the ice zone of the diagram. The cloud that forms is therefore a cloud of water ice.

In order to determine the type of nucleation involved, a study of the TP diagram is carried out. With equation (4) we can delineate two zones for the two types of nucleation, depending on the value of $r_{c}$. The different points on the graph represent the top of the cloud isotherm, where there is nucleation. Thus, we can say which type of nucleation corresponds to the cloud studied.

The red, blue, green and purple dots were samples from top boundary. Each colour corresponds to a different experimental run. That is, the time it takes for the liquid nitrogen we have poured, here each time $500 \mathrm{~g}$, to evaporate completely. So, for each experimental run, a new cloud is formed and slowly evolves.

Let $\sigma=0.09 \mathrm{~N} / \mathrm{m}[6]$ be the surface tension between ice and air, $P_{\text {sat,ice }}$ the saturating pressure of ice [7], $V_{g}=$ $3.3 \times 10^{-29} \mathrm{~m}^{3}$ the volume of a water molecule in ice, $k$ the Boltzmann constant and $r_{c}$ the critical nucleation radius. The limit for homogeneous nucleation should be one or two sizes of a water molecule, either $3.2 \AA$ or $6.4 \AA$ (see Sect. Nucleation process). These values were used as $r_{c}$ and the different nucleation zones were determined with the equation (4).

There are therefore two zones corresponding to homogeneous and heterogeneous nucleation: between the sublimation curve and the curve for $6.4 \AA$, there is heterogeneous nucleation, and below the curve for $6.4 \AA$, homogeneous nucleation becomes possible. In addition, below the dotted curve (corresponding to $r_{c}=3.2 \AA$ ), the probability of water molecules sticking upon collision tends to one, so that the vapour phase becomes unstable and fog formation rate is maximal. Be careful however, because the limit for $r_{c}=6.4 \AA$ is approximate. The boundary is not as well defined between the two types of nucleation. So we could find a homogeneous nucleation above this curve.

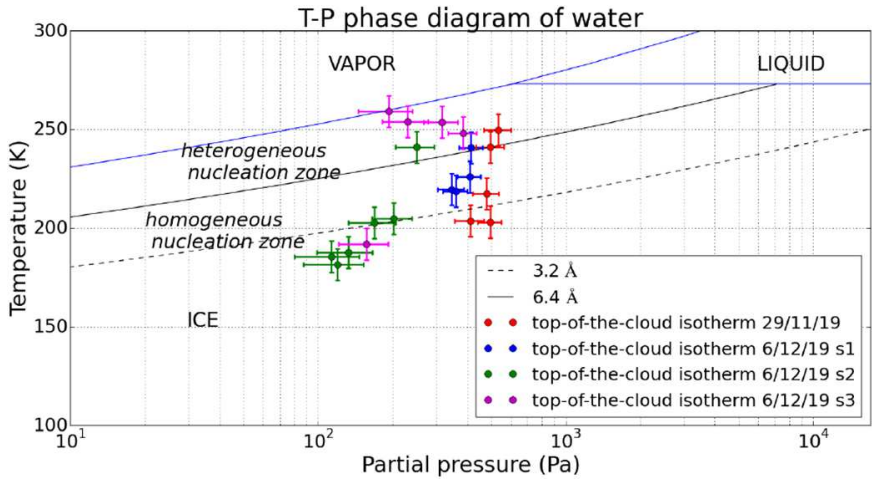

Fig. 4. TP diagram of water. The blue line marking the icevapour and liquid-vapour transitions corresponds to $P_{\text {sat }}$ as well as $\mathrm{RH}=100 \%$. The black line which separate both type of nucleation is $P_{n}$ from equation (4) with $r_{c}=6.4 \AA$. The black dotted line is $P_{n}$ for $r_{c=3.2} \AA$.

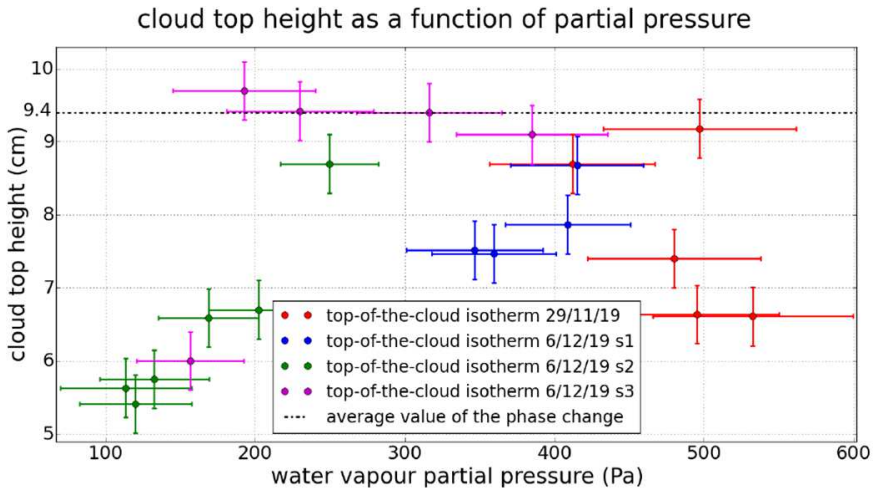

Fig. 5. Cloud top height versus the partial pressure of water vapour taken at the corresponding height with the average value of the cloud top maximal height.

Thus, it seems that both types of nucleation are present during the formation of the cloud. Indeed, the higher the cloud, the higher its temperature: heterogeneous nucleation is predominant. Conversely, the lower the cloud, the lower its temperature: homogeneous nucleation is predominant.

\subsection{Influence of the humidity on the fog top altitude}

Moisture in the box has an influence on the height of the cloud top. By checking the temperature gradient, we show that the temperature is proportional to the height. Experimentally we have obtained the following expression:

$$
T=18.140181645^{\circ} \mathrm{C} \mathrm{cm}^{-1} z-189.8669478565^{\circ} \mathrm{C}
$$

with this equation we converted the temperature into a height and obtained Figure 5. It then represents the evolution of the top of the cloud boundary as a function of the partial pressure of the water taken at the corresponding height. The graph shows that the cloud top tends to increase when the partial water pressure also increases. 
But this tendency has a limit: the purple dots seem to reach a maximum height. If we look at Figure 4, the purple dots are very close to the limit of phase change. In fact, one of them is on this limit. This explains the maximum height reached by the top of the cloud. An average value of this limit has been calculated and, in our configuration with a $30{ }^{\circ} \mathrm{C}$ plate at $11 \mathrm{~cm}$ from the nitrogen bath, we have shown experimentally that the maximum height is around $9.4 \mathrm{~cm}$. The top of the cloud cannot cross this limit of phase change because nucleation could not take place, so the cloud could not form. Thus it is the maximum height that the cloud top can reach.

\subsection{Importance of humidity}

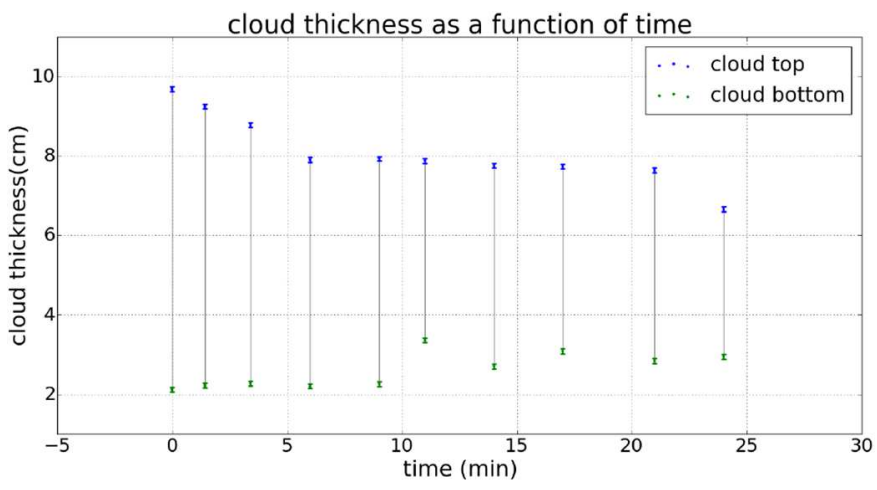

Fig. 6. Thickness of the cloud over time.

The partial water pressure also affects the thickness of the cloud. By setting the temperature of the plate at $T=30^{\circ} \mathrm{C}$, and by cutting off the supply of humid air (ergo by stopping the maintenance of constant humidity), we observe that the thickness of the cloud decreases over time (see Fig. 6). On the contrary, if the air supply is kept humid, the cloud remains unchanged until the nitrogen has completely evaporated. This shows that we do not observe a simple phase equilibrium between vapour and fog, but a dynamic process with a net water flux from vapour to fog.

The thickness and transparency of the clouds depend on the amount of water in the air, we see that the two types of cloud (in band and extended) are linked. In fact, they only differ in the amount of water vapour: an extended cloud for high humidity and a banded cloud for low humidity. This highlights that there is a critical humidity from which the cloud switches from one shape to another.

\subsection{Sources of error}

The experiment was quite difficult to carry out, so there are many sources of error. When we checked the temperature gradient, there were errors due to the device and due to the position of the thermal probe because it was difficult for the experimenter to leave the probe stationary during the time of the reading, but the latter was not very quantifiable. For the pressure measurement, the errors came from the hygrometer, the thermal probe, the diameter of the needle with which we took the air in the box and the reading of the height of the needle in the box. There is also a systematic error in the measured pressure. It is slightly higher than the actual pressure due to the vaporisation of the ice crystals from the cloud which could be taken with the air sample, once at room temperature. Finally, the error in measuring the temperature at which the sample is taken from the box was also due to the diameter of the needle and the accuracy of the previous temperature gradient.

\section{Discussion}

\subsection{Formation of the lower boundary}

For the formation of the lower boundary, we propose the following explanation. When there is a lot of humidity within the box, the drops that form are large enough to counteract the evaporation flow of nitrogen. The weight of the drops allows them to fall into the liquid nitrogen bath. So we have an extended cloud. But as the humidity decreases, the drops become smaller and smaller because there is less and less water to form the drops. There comes a humidity, called critical humidity, where the weight of the drop can no longer counteract the flow of gaseous nitrogen. When it happens the lower boundary appears (see Fig. 7).

We can calculate in our case the approximate size of the drop when this happens. In our box, $500 \mathrm{~g}$ of liquid nitrogen takes $25 \mathrm{~min}$ to evaporate completely. This corresponds to $n=10 \mathrm{mmol}$ of nitrogen gas leaving the liquid every second. With the perfect gas equation, we can find out what volume this corresponds to:

$$
V=\frac{n R T}{P}
$$

with $T$ the boiling temperature of nitrogen, $P$ is the atmospheric pressure and $R$ is the perfect gas constant. We find $V=60 \mathrm{~cm}^{3}$ of nitrogen evaporating every second. The surface area of our bath is $0.06 \mathrm{~m}^{2}$. Neglecting the fact that the interface of liquid nitrogen decreases with time, the ascending speed of gaseous nitrogen at the bath's surface is $U=1 \mathrm{~mm} \mathrm{~s}^{-1}$. Now we can equalise the weight of the drop and its viscous friction in the air [8] to determine its sedimentation speed:

$$
\frac{4}{3} \pi r^{3} \rho g=6 \pi \eta r V_{\mathrm{drop}}
$$

The boundary appears when the drop's falling speed is equal to the evaporation speed of the nitrogen gas:

$$
r^{2}=\frac{9}{2} \frac{\eta}{\rho g} U
$$




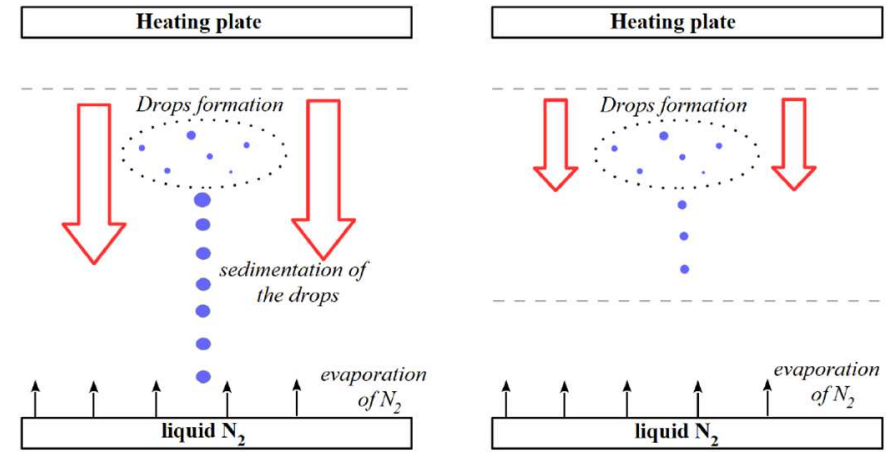

Fig. 7. scheme showing the formation of the lower boundary.

with $\rho=10^{3} \mathrm{~kg} / \mathrm{m}^{3}$ the density of the ice (neglecting air density), $\eta=10^{-5} \mathrm{Pas}$ assuming that the viscosity does not vary too much with temperature and $g=9.81 \mathrm{~m} \mathrm{~s}^{-2}$ the gravitational acceleration. We find that the radius of appearance of a banded fog whose lower limit borders on liquid nitrogen is about $2 \mu \mathrm{m}$. If the relative humidity decreases so that the radius of the crystal becomes less than $2 \mu \mathrm{m}$, two complementary phenomena could occur. Firstly, as the nitrogen gas rises, its velocity decreases because part of the nitrogen flow leaks through the slit we have made for light. As the upward velocity of nitrogen decreases with height, equation (13) shows that the radius needed to reach equilibrium also decreases. Therefore, the less moisture in the box, the higher the lower limit of the cloud should be. Secondly, if an ice crystal falls into the nitrogen despite everything, as it is a dry atmosphere, the crystal should sublimate very quickly, the smaller the radius.

\subsection{Further study}

After several months of studying this phenomenon, there is still a lot of work to be done to hope to fully understand it. Some leads can be explored:

- Checking more quantitatively whether the height of the cloud follows the equation (8) for both heterogeneous and homogeneous nucleation. For this, our experimental setup will have to be improved to allow an easier measurement of the partial pressure. And thus with enough experimental points, the verification will be done by plotting the theoretical equation on the experimental data.

- Determining a model between the nitrogen flux and the lower boundary of the cloud. This will require hydrodynamic models of the diverging nitrogen flow. These models will have to take into account the effect of gravity and buoyancy.

- Determining a model of the influence of a non-linear temperature gradient, nitrogen flow and large scale convection to see if other cloud shapes and type can be obtained.

- Determine the effects that added turbulence would have on the cloud. This could be done with the supply of humid air. For example, with this system, we could model the effect of wind on the cloud by changing the arrival speed of the moist air.

\section{Dead end}

\subsection{Ice}

We firstly used a single window for observation but it was quickly obstructed by frost formation. We have therefore replaced it by a double glazing in order to provide thermal insulation for the window. The two plexiglass panes were separated by a circulation of pressurized dry air. Second, the end of the pipe transferring the humid air was often obstructed because of the very cold atmosphere in the box; the water vapour froze in it and blocked the supply of humid air. We used a hair dryer to defrost the extremity of the pipe whenever necessary. Without forgetting that the holes made in the box could be a possible source of heat loss.

\subsection{Partial pressure measurement}

The hygrometer used could not measure relative humidity below $-20^{\circ} \mathrm{C}$, whereas the majority of our measurements would be below this temperature.

To measure the relative humidity at the desired height, it was therefore necessary to take the measurement outside the box. To this end, we first used a syringe in which we placed the hygrometer. We pierced the back wall of the box with the needle of this syringe at the required height to take the sample. The air was consequently collected into the syringe so as to measure its moisture content at ambient temperature. With this process, leaks occurred. We tried then to plug the leaks by putting a cellophane paper around both the syringe and the hygrometer to avoid and prevent leakages, but the problem persisted. Hence, we replaced this system by the vacuum pump (see The experimental setup ), which we finally used the same way we used the syringe. Since this process was successful, we used it throughout all the experiments to measure relative humidity.

\section{Conclusion}

After confirming that water vapour plays a crucial role in the formation of the cloud, we have shown that it governs both the height and the thickness of the cloud. The cloud would a priori result from two types of nucleation, depending on the pressure and temperature conditions at the time of formation. Our models and experiments show that the cloud is regulated in height by thermodynamic and hydrodynamic phenomena. Indeed, the high border of the cloud is limited by the phase transition boundary of water and results from nucleation conditions, while the lower border results presumably from a dynamic balance between the weight of the ice particles and the slowing upward flow of nitrogen.

The inverted temperature gradient and nitrogen flux imply a totally different explanation of the lower limit of 
our cloud, which corresponds to the upper limit of the Earth's atmospheric cloud. The latter is determined by the encounter of another layer of air that will block the cloud below. However, for the upper limit of our cloud and the lower limit of an atmospheric cloud, the explanation is the same. They are due to the isotherm between the gaseous state and the icy or liquid state of water. In addition, compared to the usual cloud, the temperature at the top of our cloud can drop very low, down to $-92^{\circ} \mathrm{C}$. However, our clouds could be compared to the methane clouds on Titan. Indeed, the atmosphere on Titan is mainly composed of nitrogen and has a temperature between $72 \mathrm{~K}$ and $182 \mathrm{~K}$. These temperatures allow a methane cycle similar to that of water on Earth in which methane comes from cryovolcanism. The clouds form at a height where the temperature gradient is in the same direction as our experiment [9].

We would like to thank IPT 2020 for suggesting this issue, our supervisors Adrian Daerr and Michael Berhanu, as well as the supervisors of experimental physics projects course, for their help and guidance throughout these months of research. This work would not have been possible without the assistance of our lab technician Wladimir Toutain who helped us design the experimental device for our clouds.

\section{References}

1. R. Jaenicke, Dynamics and Chemistry of Hydrometeors: Final Report of the Collaborative Research Centre 233 "Dynamik und Chemie der Meteore", 2001

2. D.E. Rosner, M. Epstein, Fog formation conditions near cool surfaces, J. Colloid Interface Sci. 28, 60-65 (1968)

3. W. Cotton, G. Bryan, S. van den Heever, Storm and Cloud Dynamics (2010)

4. H.R. Pruppacher, J.D. Klett, Microphysics of clouds and precipitation (2010)

5. A.C. Zettlemoyer, Nucleation (1997)

6. Y.S. Djikaev, E. Ruckenstein, Self-consistent determination of the ice-air interfacial tension and ice-water-air line tension from experiments on the freezing of water droplets, J. Phys. Chem. 121, 16432-16439 (2017)

7. D.M. Murphy, T. Koop, Review of the vapour pressures of ice and supercooled water for atmospheric applications, $Q . J$. R. Meteorol. Soc. 131, 1539-1565 (2005)

8. E. Guyon, J. Hulin, L. Petit, Hydrodynamique physique 3e Edition (2012)

9. F.M. Flasar, The composition of Titans atmosphere: a meteorological perspective, Planet. Space Sci. 46, 1109-1124 (1998)

Cite this article as: A. Feasson, A. Taihi, B. Correge. Two types of fog shapes above a cold evaporating liquid, Emergent Scientist 5, 2 (2021) 